Immune checkpoints are new targets for manipulation of immunological control over malignant tumors. They provide an important means to manage especially recurrent and refractory cancers and those cancers where there is an unmet need such as recurrent melanoma, renal cell carcinoma and recurrent ovarian cancer. As a new development this subject is experiencing rapid progress and multiple avenues are opening up. However, there are many hurdles to overcome, requiring constant updating, especially for students of ovarian cancer, who are looking at it with much hope.

Key words: immune checkpoint, T cell, ovarian cancer.

Contemp Oncol (Pozn) 2017; 21 (3): 189-196 DOI:https://doi.org/10.5114/wo.2017.70108

\section{Immune checkpoints, their control by immunotherapy and ovarian cancer}

\author{
Chinmoy K. Bose
}

NSC Bose Cancer Research Institute, Calcutta, India

\section{Introduction}

Immune checkpoints are many inhibitory pathways connected to the immune system that are crucial for maintaining self-tolerance. They modulate the duration and amplitude of physiological immune responses in peripheral tissues in order to minimize collateral tissue damage. The blockade of immune checkpoints has become one of the most promising approaches to activating therapeutic antitumour immunity [1]. This is T lymphocyte mediated unique tumor-specific immunity with complex pathways. The rapidity of development of drugs acting through these pathways has been spectacular. There is a great expectation from this modality in treatment. Approval has already come from the United States Food and Drug Administration (FDA) for melanomas and squamous and non-squamous non-small cell lung cancer. Ovarian cancer is another major area where immunity has been shown to play a major role. Hence, trials are underway for this indication. The initial euphoria of discovery of the drugs of the century is being replaced by the despair of the low response achieved so far. But a greater challenge awaits us, and that is to find the reason for such a stable and durable response of only about 10 to $20 \%$. It has not been possible to date to increase this even by using two or more such agents covering a broader area of mechanism of action. Currently they are combined with other targeted therapies such as PARP (poly ADP ribose polymerase) inhibitors and also with different chemotherapies. While good markers need to be developed to determine the subset of people who will benefit from this modality, extra effort seems to be needed to pinpoint the reason for such a truncated effect and more so to detect ways to increase the cure rate, subverting the weakness involved. Being a significant step towards understanding the process of neoplasia and its control, it seems worthwhile to take a close look at this subject of immune checkpoints and their use in ovarian cancer; hence this review.

\section{Major pathways}

Regulation of T lymphocyte mediated tumor-specific immunity is through highly complex pathways which include both inhibitory and stimulatory processes [2]. Cancer antigens are either released by cell death or as such present on membrane in a variety of aggressive cancer cells. Dendritic cells (DC or antigen-presenting cells, APC) recognize, take up, process, and present the tumor antigens from dead cells to the immune system. Major histocompatibility complex (MHC) class I (short peptides of 8-9 amino acids) expresses them in activated CD8+ cells while MHC class II (longer peptides of 14-20 amino acids) works by activating CD4+ T cells. Activated DC induce robust cross-priming of tumor antigen-specific $T$ cells within the draining lymph node, leading to systemic infiltration of both treated and distant tumor sites. CD8+ T cells thus migrating back to tumor sites enter the tumor microenvironment (TME). There, CD8+ T cells recognize tumor cells and lyse them, releasing additional tumor antigens and perpetuating the cycle. 
A variety of positive and negative signals can promote or hinder this process at each step. The regulation of T cell activation historically requires two signals. Signal one is transmitted after recognition of the antigen in the context of MHC by the T cell receptor (TCR). However, MHC binding is insufficient for producing a $T$ cell response by itself. In fact, lack of further stimulatory signals sends the $T$ cell into energy. Signal two is a positive signal transmitted by B7 (CD80/86) binding to CD28 balanced by a negative signal transmitted by $B 7$ binding to cytotoxic $T$ lymphocyte antigen-4 (CTLA-4) [3]. Thus, CD28 and CTLA-4 each interact with both B7-1 and B7-2. B7 is a type of peripheral membrane protein found on activated antigen-presenting cells (APC) that, when paired with either a CD28 or CD152 (CTLA-4) surface protein on a T cell, can produce a costimulatory signal or a coinhibitory signal to enhance or decrease the activity of a MHC-TCR signal between the APC and the T cell, respectively [4]. Blockade of CD28 by B7 binding is effective in stopping $T$ cell activation but CTLA-4 (CD152) has twenty times greater affinity than CD 28 for B7 proteins. Thus anti-CTLA-4 antibody is the first major immune checkpoint inhibitor that was found useful as an anticancer agent in this group. Besides being present on activated APCS, B7 is also found on T-cells themselves [5]. Cytokines can also contribute to T-cell activation, called "Signal 3". Further research has revealed an array of positive and negative regulatory signals in addition to the basic ones as described for $T$ cell activation.

\section{Other pathways}

The other most important pathway is the PD-1 (programmed cell death-1) receptor mediated pathway. It is also known as CD279 and is expressed on the surface of activated T cells, whereas its ligands, PD-L1 (B7-H1; CD274) and PD-L2 (B7-DC; CD273), are commonly expressed on the surface of dendritic cells or macrophages. PD-1 and PD-L1/PD-L2 belong to the family of immune checkpoint proteins that act as co-inhibitory factors, which can halt or limit the development of the T cell response. PD1/PD-L1 interaction ensures that the immune system is activated only at the appropriate time in order to minimize the possibility of chronic autoimmune inflammation.

When PD-L1 binds to PD-1, an inhibitory signal is transmitted into the $T$ cell, which reduces cytokine production and suppresses T-cell proliferation. Tumor cells exploit this immune-checkpoint pathway as a mechanism to evade detection and inhibit the immune response.

PD-L1 is commonly over expressed on tumor cells or on non-transformed cells in the tumor microenvironment [1]. PD-L1 expressed on the tumor cells binds to PD-1 receptors on the activated T cells, which leads to inhibition of the cytotoxic T cells. These deactivated T cells remain inhibited in the tumor microenvironment. The PD-1/PD-L1 pathway represents an adaptive immune resistance mechanism that is exerted by tumor cells in response to endogenous anti-tumor activity. In regulation of T cells by the immune checkpoint the net signal shapes the strength and quality of the T cell response.
While both CTLA4 and PD1/PD-L1 are co-inhibitory pathways used to produce drugs in such therapy, there are a number of co-stimulatory pathways such as CD28 as well which are used to discover newer drugs. Five of them are of the TNF receptor super family: CD27, CD40, OX40, GITR and CD137. Another two stimulatory checkpoint molecules belong to the B7-CD28 superfamily: CD28 itself and ICOS. Still another simulator, CD122, is the interleukin-2 receptor $\beta$ sub-unit. All of them promoted $T$ cell proliferation. CD28 was the target of the TGN1412 "superagonist" which caused severe inflammatory reactions in the first-in-man study in London in March 2006 [6].

Celldex Therapeutics is working on CDX-1127, an agonistic anti-CD27 monoclonal antibody [7] which in animal models has been shown to be effective in the context of T cell receptor stimulation [8].

CD40 - this molecule, found on a variety of immune system cells including antigen-presenting cells, has CD4OL. The Swiss pharmaceutical company Roche acquired this project of anti-CD40 agonist monoclonal antibody when VLST was shut down in 2013 [9].

CD 137, also called 4-1BB, when bound by the CD137 ligand, results in T-cell proliferation. The German biotech company Pieris Pharmaceuticals has developed an engineered lipocalin that is bi-specific for CD137 and HER2 [10].

Anti-OX40 monoclonal antibodies have been shown to have clinical utility in advanced cancer [11].

The pharmaceutical company AstraZeneca has three drugs in development targeting OX40. TG Therapeutics is working on anti-GITR antibodies which have been shown to promote an anti-tumor response through loss of Treg lineage stability [12].

Nektar Therapeutics is working on NKTR-214, a CD122based immune-stimulatory cytokine to increase proliferation of CD8+ effector T cells. Jounce Therapeutics is developing an ICOS agonist for its role in T cell effector function $[13,14]$.

Drugs or drug candidates that inhibit/block the inhibitory checkpoint molecules (above) are confusingly sometimes known as immune checkpoint inhibitors.

Chemokines promote the trafficking of $\mathrm{T}$ cells to tumors, where selectins and LFA1 or the vascular endothelial growth factor (VEGF) and the endothelin B receptor (ETBR) can promote or deter T cell infiltration into the tumor [15]. Within the tumor a variety of immune suppressive influences inhibit $T$ cell activity. These include immune suppressive cells $[$ CD4+FOXp3+ regulatory $T$ cells (Treg) and myeloid-derived suppressor cells (MDSC)], immune suppressive cytokines [transforming growth factor- $\beta$ (TGF- $\beta$ ) and interleukin 10 (IL-10)], metabolic enzymes [indoleamine 2,3-dioxygenase (IDO) and arginase], and immune checkpoints [programmed death ligand-1 (PD-L1)] [1, 16]. Tumor cells can co-opt immune checkpoint pathways to evade the T cell response, expressing PD-L1 on their surface as the result of constitutive oncogenic signaling [17], epithelial to mesenchymal transition [18] or adaptive resistance to immune attack [19]. Regardless of the mechanism, tumor cell surface PD-L1 expression provides a means of evading active immunity $[19,20]$. Developing strategies for abrogating these immune suppressive mechanisms to 
support CD8+ T cell activity at the tumor site is critical for the success of immunotherapy.

All other costimulatory molecules including antagonists of their antibodies are subject to intense research efforts by many pharmaceutical companies.

\section{Ovarian cancer scenario}

Molecular biology of ovarian cancer has offered a little to the chemo- or recent targeted therapy approach to ovarian cancer treatment so far. According to the literature, the signaling pathways associated with OVC are the following: the nuclear factor $\kappa$-light-chain-enhancer of activated B cells (NF- $\kappa \mathrm{B}$ ) pathway, the activator of transcription 3 (Jak-STAT 3) pathway, the mitogen-activated protein kinase (MAPK) pathway, the proto-oncogene tyrosine protein kinase Src pathway, the ErbB activation pathway, the lysophosphatidic acid (LPA) pathway, the phosphatidylinositol 3-kinase (PI3K) pathway, the Müllerian inhibitory substance receptor pathway, the EGF and VGEF pathways and the ER $\beta$ pathway. However, there is an unmet need to find a biomarker as well as the most suitable treatment of ovarian cancer. As a result, the possibility of immunotherapy being used here is worth looking at.

More than three decades ago, T cell infiltration was observed in ovarian cancers [21]. In 2003 Zhang et al. [22] appreciated their role in improved survival. They observed at least $60 \%$ benefit in 5 -year survival in a cohort of 74 patients who were treated well with a complete clinical response after debulking and platinum-based therapy. $\mathrm{CD}^{+} \mathrm{T}$ cells within their tumor and monokines induced by IFN- $\gamma$ plus macrophage-derived chemokines made the difference [22]. The reason for such heterogeneity is still unclear, though the subject has grown and gained much strength. While regulatory T-cell subsets (CD4+) have a confusing immune suppressing role as regulatory $T$ cells, improved survival was noted in patients who had higher numbers of intraepithelial CD8+ T cells compared with patients without intraepithelial CD8+ T cells (median survival 55 vs. 26 months) [23, 24]. A strong positive correlation is observed between levels of CD8 + T cells and granzyme B within tumors [25]. MHC-related interferon regulatory factor (IRF)-1 and metastasis-related chemokine receptor (CXCR)6 are two genes differentially expressed in tumors with high versus low CD8+ T-cell infiltration. Heterogeneity in the tumor microenvironment among patients with epithelial ovarian cancer (EOC) and various immune cell populations of those have been associated positively or negatively with clinical prognosis, including tumor-infiltrating lymphocytes (TILS) [22, 23, 26], myeloid-derived suppressor cells [27], and tumor-associated macrophages [28]. TILs express the negative regulatory immune receptor programmed cell death 1 (PD-1) [29] which is upregulated on T-cell activation and suppresses T-effector functions, whereas several cellular populations, including cancer cells and tumor-associated myeloid cells, express its ligand PD-L1 [30-33]. Expression of PD-L1 by tumors has been associated with decreased intraepithelial TILs and poor overall survival in $\mathrm{EOC}$ [33].
In an immune-competent murine model of EOC, PD-1 and PD-L1 blockade has led to eradication of tumors through the expected reprogramming of the tumor microenvironment [34], which suggests potential benefit from PD-1/PD-L1 inhibition for patients with EOC. The attenuation of $T$ cell function by cytotoxic $T$ lymphocyte-associated antigen 4 (CTLA-4) was also noted in EOC [35]. Although the binding of B7-1 or B7-2 to CD28 provides an important costimulatory signal, the engagement of CTLA- 4 by these ligands induces cell cycle arrest and diminished cytokine production [36-38].

Documented effort to test ovarian cancer started with Hodi et al. [39], who gave two patients a single infusion and then nine cases [40] up to 11 infusion of $3 \mathrm{mg} / \mathrm{kg}$ ipilimumab after an autologous ovarian tumor cell vaccine transduced with granulocyte-macrophage colony-stimulating factor (GVAX). Only three patients had SD of $>2$ months. A phase 2 clinical trial of ipilimumab in relapsed platinum-sensitive ovarian cancer with measurable disease is ongoing though not recruiting any more (NCT01611558). Some trials either in post-transplantation or along with other checkpoint molecules such as nivolum$a b$ and pembrolizumab are recruiting. There are two other trials which are either suspended or terminated. The PD-1 antagonist nivolumab is being tested at 1 or $3 \mathrm{mg} / \mathrm{kg}$ every 2 weeks in 18 patients with relapsed platinum-resistant disease regardless of PD-L1 expression; there was a 17\% overall response rate (ORR) and a 44\% disease control rate $(D C R=C R+P R+S D)$, with $2 C R, 1 P R$, and 5 patients with $\mathrm{SD}$ [41]. A phase $1 \mathrm{~b}$ study tested the PD-1 antagonist pembrolizumab at $10 \mathrm{mg} / \mathrm{kg}$ every 2 weeks in 26 patients with heavily treated PD-L1+ ovarian cancer chemotherapy [42]. There was a durable ORR of $11.5 \%$, and a DCR of $34.6 \%$, with 1 CR, 2 PR, and 6 patients with SD. Now pembrolizumab has the highest number of clinical trials in ovarian cancer. The PD-L1 antagonist avelumab was given at $10 \mathrm{mg} / \mathrm{kg}$ every 2 weeks in a phase $1 \mathrm{~b}$ study of 75 patients with platinum-resistant or chemotherapy-refractory ovarian cancer regardless of PD-L1 expression [43] with an ORR of $10.7 \%$, and a DCR of $54.7 \%$. A phase 1 study of another PD-L1 antagonist, BMS-936559, revealed one objective response in 17 ovarian cancer patients [44]. It is evident from the discussion that many obstacles are remaining in establishing immunotherapy in ovarian cancer as the response rate is low and no FDA approval is in the offing. As expected, Professor Maurie Markman commented at the $33^{\text {rd }}$ Annual Chemotherapy Foundation Symposium "The checkpoint inhibitors are not ready for prime time yet in ovarian cancer. It's not because there's evidence that they don't work - it's just that there's no evidence at all".

\section{New drugs of immunotherapy}

\section{Anti-CTLA-4 Ab}

\section{IgG 1 isotype - ipilimumab}

The anti-CTLA-4 antibody (Ab) (previously, MDX-010; provided by Medarex) is a fully human $\operatorname{lgG}_{1 \kappa} \mathrm{Ab}$ derived from transgenic mice having human genes encoding heavy and light chains to generate a functional human repertoire. This Ab has been shown to bind to CTLA-4 ex- 
pressed on the surface of human T cells and inhibit binding of CTLA-4 to B7 molecules [45]. It is the first anti-CTLA4 agent in clinical development. It was approved by the FDA in 2011 and the European Medicines Agency for the treatment of metastatic melanoma following research showing improved survival [46]. I pilimumab antagonizes CTLA-4 and prevents ligand binding [47]. It is undergoing clinical trials for the treatment of non-small cell lung carcinoma (NSCLC), small cell lung cancer (SCLC) [48], bladder cancer [49] and metastatic hormone-refractory prostate cancer [50].

Brunet et al. [51] of Institut national de la santé et de la recherche médicale, France (Inserm) came across cDNA clones defining a sequence, CTLA-4, which could encode a 223-amino-acid protein while screening mouse cytolytic-T-cell-derived cDNA libraries. Jim Allison of Berkley with graduate student Max Krummel and postdoctoral fellow Cynthia Chamber were able to provide evidence that CTLA4 actually served to inhibit the activity of $T$ cells. That was the beginning. They performed an experiment in 6-weekold golden Syrian hamsters who received five footpad injections of heat-killed Staphylococcus A bacteria coated with CTLA-4lg and suspended in $0.2 \mathrm{ml}$ of PBS. Three days after the final injection, draining lymph nodes were removed, and lymphocytes were isolated and fused with the P3X3.Ag8.653 myeloma line using a standard polyethylene glycol fusion technique. Hybridoma supernatants were tested for reactivity to CTLA-4Ig and for a lack of reactivity to CD4 Ig by ELISA, thus developing the first anti-CTLA-4 antibody [52]. Medarex (former NASDAQ symbol: MEDX), an American biopharmaceutical company based in Princeton, New Jersey, developed an anti-CTLA-4 monoclonal antibody, MDX-010. In 2009, Medarex was purchased by Bristol Myers Squibb.

\section{$\operatorname{IgG} 2$ isotype, tremelimumab}

Tremelimumab (formerly CP-675,206) is a human IgG2 monoclonal antibody specific for CTLA4. In a phase III trial in advanced melanoma, 655 patients were enrolled and randomly assigned to treatment with tremelimumab or chemotherapy. Previously in development by Pfizer [53], it is now in investigation by Medlmmune, a wholly owned subsidiary of AstraZeneca [54]. Unlike ipilimumab, which is an IgG1 isotype, tremelimumab is an IgG2 isotype [55] and has not attained approval so far.

\section{PD-1 \& PD-L1-targeting agents \\ $P D-1$}

Nivolumab (ONO-4538, BMS-936558, or MDX1106), marketed as Opdivo, is a human IgG4 anti-PD-1 monoclonal antibody developed by Ono Pharmaceutical and Medarex (later acquired by Bristol-Myers Squibb) for the treatment of cancer. It is a fully human IgG4 monoclonal antibody targeting PD-1. It is approved by the FDA for treatment of patients with unresectable or metastatic melanoma who no longer respond to other drugs (December 2014). In addition, it is approved for the treatment of squamous non-small cell lung cancer (March 2015). Patients in the trial had advanced melanoma, NSCLC, castration-resistant prostate cancer, renal cell carcinoma (RCC), or col- orectal cancer (CRC). Patients received nivolumab at doses of 0.1 to $10.0 \mathrm{mg} / \mathrm{kg}$ of body weight every 2 weeks for up to 12 cycles until disease progression or a complete response occurred.

Pembrolizumab: On September 4, 2014 the FDA approved pembrolizumab under the FDA Fast Track Development Program [56]. It is approved for use following treatment with ipilimumab, or after treatment with ipilimumab and a BRAF inhibitor in advanced melanoma patients who carry a BRAF mutation [57]. It is marketed by Merck. Pembrolizumab (MK-3475, formerly lambrolizumab) has been very successful in treating melanoma and NSCLC, similarly to nivolumab. Significant differences cannot be assessed in the absence of a randomized trial comparing the two agents. However, binding affinities of the agents are different. In phase I trials, neither agent has been found to have a maximally tolerated dose. That said, more time and energy has been spent on searching for an appropriate dose for pembrolizumab. Pembrolizumab was invented by Gregory Carven, Hans van Eenennaam and John Dulos at Organon Biosciences, which later became Schering Plough Research Institute and then Merck \& Co [58]. MRC Technology humanized the antibody pembrolizumab for Organon in 2016.

On October 2, 2015, the FDA approved pembrolizumab for the treatment of metastatic non-small cell lung cancer in patients whose tumors express PD-L1 and who have failed treatment with other chemotherapeutic agents.

Pidilizumab is a humanized IgG1 antibody targeting PD-1. The agent was initially evaluated in a phase I trial targeting hematologic malignancies. Presently, there are a number of clinical trials underway in both hematologic and solid tumors [59].

The results of two pidilizumab clinical trials were recently published in peer-reviewed journals. In a single-center, single-arm, phase II trial, 32 patients with relapsed follicular lymphoma received pidilizumab at a dose of $3 \mathrm{mg} / \mathrm{kg}$ every 4 weeks for 4 infusions with up to 8 additional infusions administered. In addition, rituximab was given at a dose of 375 $\mathrm{mg} / \mathrm{m}^{2}$ of body surface area every week for 4 weeks. Investigators reported that 19 of 29 evaluable patients achieved an objective response, with complete responses in 15 patients (51.7\%) [60].

An additional phase II trial involved patients with diffuse large B-cell lymphoma (DLBCL) following autologous hematologic stem cell transplantation (AHSCT). Sixty-six patients were treated with 3 doses of pidilizumab in the first 1 to 3 months after AHSCT. The progression-free survival rate was $72 \%$ at 6 months after AHSCT $(90 \% \mathrm{Cl}, 60 \%$ to $82 \%)$, meeting the primary endpoint. Thirty-five patients had measurable disease following AHSCT, and the response rate in those patients was 51\% [61].

\section{$P D$-L1-targeting agents}

PD-L1 inhibitors are currently undergoing clinical trials for treatment of various types of cancer; however, no PD-L1 inhibitors have since been approved by the FDA.

Atezolizumab: MPDL3280A is an engineered human IgG1 monoclonal antibody that targets PD-L1. Combin- 
ing unique property to eliminate antibody-dependent cell-mediated cytotoxicity (ADCC) effector function, MPDL3280A, unlike some other anti-PD-L1 antibodies, does not deplete cells expressing PD-L1. It appears to have significant activity in a subset of patients with NSCLC. This was demonstrated in a phase I trial of atezolizumab in which 53 patients with NSCLC had a $23 \%$ response rate where PD-L1 expression is shown; thus PD-L1 might even be an immunohistochemical biomarker with some degree of predictive capability. The POPLAR trial compared atezolizumab with docetaxel in the second- or third-line setting in patients with NSCLC, regardless of histology, where the OS benefit with atezolizumab compared with docetaxel in patients with a high level of PD-L1 expression.

Durvalumab: MEDI4736 is a human IgG1 monoclonal antibody recognizing human PD-L1. It is similar to MPDL3280A in eliminating complement-mediated cytotoxicity and ADCC due to mutations in the Fc receptor. A phase I dose of $10 \mathrm{mg} / \mathrm{kg}$ every 2 weeks is currently being evaluated in several histologies in an expansion phase [62]. Brahmer et al. treated 13 NSCLC patients with MEDI4736, with 3 partial responses [63] as presented in the 2014 ASCO Annual Meeting. Several large combination trials in lung cancers are ongoing. Presently 5 trials are aimed at ovarian cancer.

Avelumab: MSB0010718C is a fully human monoclonal PD-L1 antibody of isotype IgG1, currently in development by Merck KGaA, Darmstadt, Germany \& Pfizer for NSCLC [64]. MSB0010718C is an IgG1 targeting PD-L1. It is a native Fc receptor, allowing for antibody-dependent cell-mediated cytotoxicity. Avelumab binds to PD L1 and inhibits binding (PD-1). Formation of a PD-1/PD-L1 receptor/ligand complex is prevented, leading to an increased CD8+ T cell mediated immune response [65].

BMS-936559: BMS-936559 or MDX-1105 is a high-affinity, fully human IgG4 monoclonal antibody that binds PDL1 and that blocks PD-L1 from binding its two known receptors, PD-1 and CD8 $[66,67]$. It was found safe in a phase I trial that included 17 ovarian cancer patients in escalating doses of $0.3-10 \mathrm{mg} / \mathrm{kg}$ iv every 14 days in 6-week cycles for up to 16 cycles and achieved objective responses: 1 (6\%) with a partial response and $3(18 \%)$ with stable disease lasting more than 24 weeks. Common side effects included fatigue, infusion reactions, diarrhea, arthralgia, pruritus, rash, nausea, and headache.

The drawbacks associated with antibodies include their large size and their ability to activate antibody dependent cell-mediated cytotoxicity, through their Fc-region. While Fc-mediated effects are an important part of the efficacy of many antibody therapeutics, in the case of PD-1/PD-L1 inhibition this may be counterproductive. Smaller engineered affinity proteins offer a potentially more effective alternative to antibody therapeutics as PD-L1 inhibitors.

As an example, Avacta Life Sciences are developing a PD-L1 inhibitor to meet this need. It is based on their engineered Affimer protein scaffolds. The use of a smaller inhibitor should improve diffusion of the therapeutic throughout solid tumors. Multimeric Affimer biotherapeutic formats can also be engineered to create bi-specific molecules that combine two different immune checkpoint inhibitors to improve efficacy and offer a better clinical outcome for the patient

\section{Other drugs}

Enoblituzumab is a monoclonal antibody designed for the treatment of cancer. Formerly known as MGA271, the drug is a humanized IgG1к monoclonal antibody recognizing human $\mathrm{B} 7-\mathrm{H} 3$.

Varlilumab is a fully human monoclonal agonist anti-CD27 monoclonal antibody (mAb) that has been shown to activate human $T$ cells in the context of $T$ cell receptor stimulation, with potent anti-tumor responses. It may be particularly effective in combination with other immunotherapies. In addition to the immune-enhancing properties of varlilumab, the mAb may also provide direct therapeutic effects against tumors with CD27 expression. Human $B$ and $T$ cell lymphomas often express CD27 at high levels, and varlilumab has shown potent anti-tumor activity against these types of tumors in preclinical models. Therefore, in patients with lymphomas/leukemia that express CD27, varlilumab may function through two independent mechanisms.

Lirilumab (INN) is a human monoclonal antibody designed to bind to KIR 2DL1/2L3 [68]. This drug was developed by Innate Pharma and is licensed to Bristol-Myers Squib.

Epacadostat is an orally available hydroxyamidine. It inhibits indoleamine 2,3-dioxygenase (IDO), an enzyme responsible for the oxidation of tryptophan into kynurenine, with potential immunomodulating and antineoplastic activities. Epacadostat targets and binds to IDO, an enzyme responsible for the oxidation of tryptophan into kynurenine. Thus it increases and restores the proliferation and activation of various immune cells, including dendritic cells (DCs), NK cells, and T-lymphocytes, as well as interferon (IFN) production, and a reduction in tumor-associated regulatory $T$ cells (Tregs).

\section{Toxicities of immunotherapy}

Although these agents can lead to remarkable responses, their use can also be associated with unique immune-related adverse effects (irAEs). In general, use of PD-1 inhibitors such as nivolumab and pembrolizumab has a lower incidence of irAEs compared with those that block CTLA-4 such as ipilimumab. The combination of nivolumab and ipilimumab has a higher rate of irAEs than either approach as monotherapy. Consensus guidelines regarding the treatment of the most common irAEs including rash, colitis, hepatitis, endocrinopathies, and pneumonitis have been established. The mainstay of irAE treatment consists of immunosuppression with corticosteroids or other immunosuppressant agents such as infliximab; most irAEs will resolve with appropriate management.

\section{Combinations of immunotherapeutic agents}

Combinations can be of three types where either a) two or three immune checkpoint inhibitors are used, b) other targeted therapy is used or c) chemotherapy and other standard treatments are used. Other than the promising 
single agent indications mentioned above, immune checkpoint inhibition is not effective in a majority of patients. Newer combination strategies are planned and executed. In one such trial, tremelimumab and interferon were administered concurrently in standard doses, followed by maintenance interferon. Of the 33 evaluable patients, there were 3 complete responses and 7 partial responses. The median OS was 15.9 months [69]. One area of remarkable success involved the use of nivolumab and ipilimum$a b$ in patients with metastatic melanoma. A randomized phase II trial of ipilimumab investigated the potential synergy of adding granulocyte macrophage colony-stimulating factor (GM-CSF, or sargramostim) with ipilimumab [70]. The response rate was 53\%. The combination of ipilimumab and nivolumab has subsequently been evaluated in a variety of malignancies. In RCC, two variations of the combination were tested. The same regimen was evaluated in NSCLC, with disappointing results. Carefully tailored regimens for specific patient populations and lower doses are planned now ( $1 \mathrm{mg} / \mathrm{kg}$ of ipilimumab and $1 \mathrm{mg} / \mathrm{kg}$ of nivolumab [71].

Among recent combinations used in advanced and refractory ovarian cancer, a safety study has been started for ipilimumab in combination with MGA271 (enoblituzumab). A Phase 1/2, Open-label Study is recruiting where nivolumab monotherapy or nivolumab combined with ipilimumab is used in subjects with advanced or metastatic solid tumors. Tremelimumab is combined with a PARP inhibitor in BRCA-deficient ovarian cancer and also with either durvalumab or durvalumab plus first-line chemotherapy in advanced solid tumors including ovarian cancer.

A study of the safety, tolerability, and efficacy of epacadostat administered in combination with nivolumab and a dose escalation and cohort expansion study of anti-CD27 (varlilumab) and anti-PD-1 (nivolumab) in select advanced cancers includes ovarian cancer patients.

Pembrolizumab is becoming very popular as at least seventeen trials are planned or recruiting in combination with MGA271, epacadostat, niraparib, acalabrutinib (ACP196), colony stimulating factor 1 receptor inhibitor PLX3397 (pexidartinib), VEGF inhibitor ziv-aflibercept, PEGylated recombinant human IL-10 (AM0010), and standard chemotherapy in ovarian cancer.

Avelumab in combination with pegylated liposomal doxorubicin is starting. Durvalumab and pembrolizumab with tremelimumab or VTX-2337 (a novel Toll-like receptor, TLR 8 agonist), with olaparib or cediranib is being tried. Recently atezolizumab plus bevacizumab with or without acetylsalicylic acid is being tried in a recurrent platinum-resistant ovarian cancer trial.

\section{Combining chemotherapy and immunotherapy}

The discovery of crucial molecular pathways that promote tumor growth and maintenance together with the development of drugs that specifically inhibit these pathways has ushered in a new era of cancer medicine [72]. Analogously, an improved understanding of the mechanisms of protective anti-tumor immunity and the translation of these concepts into efficacious immuno- therapies that prolong patient survival has validated the long-standing idea that immunity plays an important role in cancer pathogenesis. The complementary modes of action of these two promising modalities suggest intriguing possibilities for therapeutic synergy with combination treatment. A strong foundation has been established to advance the crafting of both preclinical and early stage patient investigations to determine the best ways to integrate targeted agents and immunotherapy.

In conclusion, handling immunological therapy has never been very smooth. The glaring mechanism of action and efficacy was a breakthrough of the year in 2013, which was true at least for melanoma [73]. The subject being very interesting, hard work is needed to find out what more is needed to achieve an even better clinical effect. But before that translational scientists and clinical investigators should address efficiently a variety of important clinical and scientific questions regarding the tumor microenvironment and interactions of tumor biology with human immunology. As combinations have already been tried and have shown promise, experience and experimentation with these drugs may lead to a bigger breakthrough if not a medical breakthrough of the century.

The author declares no conflict of interest.

\section{References}

1. Pardoll DM. The blockade of immune checkpoints in cancer immunotherapy. Nat Rev Cancer 2012; 12: 252-64.

2. Chen DS, Mellman I. Oncology meets immunology: the cancer-immunity cycle. Immunity 2013; 39: 1-10.

3. Lenschow DJ, Walunas TL, Bluestone JA. CD28/B7 system of T cell costimulation. Annu Rev Immunol 1996; 14: 233-58.

4. Coico R, Sunshine G. Eli Benjamini. Immunology: A Short Course. Wiley-Liss 2003; 131.

5. Taylor PA, Lees CJ, Fournier S, Allison JP, Sharpe AH, Blazer BR. B7 expression on $\mathrm{T}$ cells down-regulates immune responses through CTLA-4 ligation via T-T interactions. J Immunol 2004; 172: 34-9.

6. Eastwood D, Findlay L, Poole S, et al. Monoclonal antibody TGN1412 trial failure explained by species differences in CD28 expression on CD4+ effector memory T-cells. Br J Pharmacol 2010; 161: 512-26.

7. CDX-1127 - Monoclonal Antibody Targeting CD27. Celldex Therapeutics.

8. He LZ, Prostak N, Thomas LJ, et al. Agonist anti-human CD27 monoclonal antibody induces $T$ cell activation and tumor immunity in human CD27-transgenic mice. J Immunol 2013; 191: 4174-83.

9. Zimm A. Cancer 'Miracle' Patients Studied Anew for Disease Clues. Bloomberg. Retrieved 25 June 2015.

10. Pieris Pharmaceuticals to present data on novel anti-CD137 and HER2 bispecificimmuno-oncology program at UBS Global Healthcare Conference". Pieris Pharmaceuticals. 19 May 2015. Retrieved 5 June 2015

11. Curti BD, Kovacsovics-Bankowski M, Morris N, et al. OX40 is a potent immune-stimulating target in late-stage cancer patients. Cancer Res 2013; 73: 7189-98.

12. Schaer DA, Budhu S, Liu C, et al. GITR pathway activation abrogates tumor immune suppression through loss of regulatory $T$ cell lineage stability. Cancer Immunol Res 2013; 1: 320-31.

13. Disrupting Tumor Evasion of the Immune System. NewLink Genetics. Retrieved 5 June 2015.

14. Philips GK, Atkins M. Therapeutic uses of anti-PD-1 and anti-PD-L1 antibodies. IntImmunol 2015; 27: 39-46. 
15. Buckanovich RJ, Facciabene A, Kim S, et al. Endothelin B receptor mediates the endothelial barrier to $T$ cell homing to tumors and disables immune therapy. Nat Med 2008; 14: 28-36.

16. Balkwill F, Mantovani A. Inflammation and cancer: back to Virchow? Lancet 2001; 357: 539-545.

17. Akbay EA, Koyama S, Carretero J, et al. Activation of the PD-1 path way contributes to immune escape in EGFR-driven lung tumors. Cancer Discov 2013; 3: 1355-63.

18. Chen L, Gibbons DL, Goswami S, Cortez MA, Ahn YH, Byers LA, Zhang X, Yi X, Dwyer D, Lin W, Diao L, Wang J, Roybal JD, Patel M, Ungewiss C, Peng D, Antonia S, Mediavilla-Varela M, Robertson G, Jones S, et al. Metastasis is regulated via microRNA-200/ZEB1 axis control of tumour cell PD-L1 expression and intratumoral immunosuppression. Nat Commun 2014; 5: 5241.

19. Topalian SL, Hodi FS, Brahmer JR, et al. Safety, activity, and immune correlates of anti-PD-1 antibody in cancer. N Engl J Med 2012; 366: 2443-54.

20. Barber DL, Wherry EJ, Masopust D, Zhu B, Allison JP, Sharpe AH, Freeman GJ, Ahmed R. Restoring function in exhausted CD8 T cells during chronic viral infection. Nature 2006; 439: 682-7.

21. Haskill S, Becker S, Fowler W, et al. Mononuclear-cell infiltration in ovarian cancer. I. Inflammatory-cell infiltrates from tumour and ascites material. Br J Cancer 1982; 45: 728-36.

22. Zhang L, Conejo-Garcia JR, Katsaros D, et al. Intratumoral T cells, recurrence, and survival in epithelial ovarian cancer. N Engl J Med 2003; 348: 203-13.

23. Sato E, Olson SH, Ahn J, et al. Intraepithelial CD8+ tumor-infiltrat ing lymphocytes and a high CD8+/regulatory $T$ cell ratio are associated with favorable prognosis in ovarian cancer. Proc Natl Acad Sci USA 2005; 102: 18538-43.

24. Leffers N, Vermeij R, Hoogeboom BN, et al. Long-term clinical and immunological effects of p53-SLP ${ }^{\circledR}$ vaccine in patients with ovarian cancer. Int J Cancer 2012; 130: 105-12.

25. Milne K, Kobel M, Kalloger SE, et al. Systematic analysis of immune infiltrates in high-grade serous ovarian cancer reveals CD20, FoxP3 and TIA-1 as positive prognostic factors. PLoS One 2009; 4: E6412.

26. Curiel TJ, Coukos G, Zou L, et al. Specific recruitment of regulatory $T$ cells in ovarian carcinoma fosters immune privilege and predicts reduced survival. Nat Med 2004; 10: 942-9.

27. Cho H, Hur HW, Kim SW, et al. Pre-treatment neutrophil to lymphocyte ratio is elevated in epithelial ovarian cancer and predicts survival after treatment. Cancer Immunol Immunother 2009; 58: $15-23$.

28. Zhang $M$, He Y, Sun X, et al. A high M1/M2 ratio of tumor-associated macrophages is associated with extended survival in ovarian cancer patients. J Ovarian Res 2013; 7: 19.

29. Matsuzaki J, Gnjatic S, Mhawech-Fauceglia P, et al. Tumor-infiltrating NY-ESO-1-specific CD8+ T cells are negatively regulated by LAG-3 and PD-1 in human ovarian cancer. Proc Natl Acad Sci USA 2010; 107: 7875-80

30. Abiko K, Mandai M, Hamanishi J, et al. PD-L1 on tumor cells is induced in ascites and promotes peritoneal dissemination of ovarian cancer through CTL dysfunction. Clin Cancer Res 2013; 19: 1363-74.

31. Liu Y, Zeng B, Zhang Z, et al. B7-H1 on myeloid-derived suppressor cells in immune suppression by a mouse model of ovarian cancer. Clin Immunol 2008; 129: 471-81.

32. Curiel TJ, Wei S, Dong $\mathrm{H}$, et al. Blockade of B7-H1 improves myeloid dendritic cell-mediated antitumor immunity. Nat Med 2003; 9: 562-7.

33. Hamanishi J, Mandai M, Iwasaki M, et al. Programmed cell death 1 ligand 1 and tumor-infiltrating CD8 T lymphocytes are prognostic factors of human ovarian cancer. Proc Natl Acad Sci USA 2007; 104: 3360-5.

34. Duraiswamy J, Freeman GJ, Coukos G. Therapeutic PD-1 pathway blockade augments with other modalities of immunotherapy T-cell function to prevent immune decline in ovarian cancer. Cancer Res 2013; 73: 6900-12.

35. Preston CC, Goode EL, Hartmann LC, Kalli KR, Knutson KL. Immunity and immune suppression in human ovarian cancer. Immunotherapy 2011; 3: 539-56.
36. Thompson CB, Allison JP. The emerging role of CTLA-4 as an immune attenuator. Immunity 1997; 7: 445-50.

37. Doyle AM, Mullen AC, Villarino AV, et al. Induction of cytotoxic T lymphocyte antigen 4 (CTLA-4) restricts clonal expansion of helper T cells. J Exp Med 2001; 194: 893-902.

38. Salomon B, Bluestone JA. Complexities of CD28/B7: CTLA-4 COstimulatory pathways in autoimmunity and transplantation. Annu Rev Immunol 2001; 19: 225-52.

39. Hodi FS, Mihm MC, Soiffer RJ, et al. Biologic activity of cytotoxic T lymphocyte-associated antigen 4 antibody blockade in previously vaccinated metastatic melanoma and ovarian carcinoma patients. Proc Natl Acad Sci U S A 2003; 100: 4712-7.

40. Hodi FS, Butler M, Oble DA, et al. Immunologic and clinical effects of antibody blockade of cytotoxic T lymphocyte-associated antigen 4 in previously vaccinated cancer patients. Proc Natl Acad Sci U S A 2008; 105: 3005-10.

41. Hamanishi J, Mandai M, Ikeda T, et al. Efficacy and safety of anti-PD-1 antibody (Nivolumab: BMS-936558, ONO-4538) in patients with platinum-resistant ovarian cancer. J Clin Oncol 2014; 32 (5 suppl): abstr \#5511.

42. Varga A, Piha-Paul SA, Ott PA, et al. Antitumor activity and safety of pembrolizumab in patients (pts) with PD-L1 positive advanced ovarian cancer: Interim results from a phase Ib study. J Clin Oncol 2015; 33 (Suppl): abstr \#5510.

43. Disis ML, Patel MR, Pant S, et al. Avelumab (MSB0010718C), an anti-PD-L1 antibody, in patients with previously treated, recurrent or refractory ovarian cancer: A phase Ib, open-label expansion trial. J Clin Oncol 2015; 33 (Suppl): abstr \#5509.

44. Brahmer JR, Tykodi SS, Chow LQ, et al. Safety and activity of anti-PD-L1 antibody in patients with advanced cancer. N Engl J Med 2012; 366: 2455-65.

45. Phan GO, Yang JC, Sherry RM, et al. Cancer regression and autoimmunity induced by cytotoxic $T$ lymphocyte-associated antigen 4 blockade in patients with metastatic melanoma. Proc Natl Acad Sci U S A 2003; 100: 8372-7.

46. Hodi FS, O’Day SJ, McDermott DF, et al. Improved survival with ipilimumab in patients with metastatic melanoma. N Engl J Med 2010; 363: 711-23.

47. Tarhini AA, Thalanayar PM. Melanoma adjuvant therapy. Hematol Oncol Clin North Am 2014; 28: 471-89.

48. Clinical trial number NCT00527735 at ClinicalTrials.gov Phase II Study for Previously Untreated Subjects With Non Small Cell Lung Cancer (NSCLC) or Small Cell Lung Cancer (SCLC).

49. First-Line Gemcitabine, Cisplatin + Ipilimumab for Metastatic Urothelial Carcinoma Clinical trial number NCT01524991 at ClinicalTrials.gov.

50. Clinical trial number NCT00323882 at ClinicalTrials.gov Phase I/II Study of MDX-010 in Patients With Metastatic Hormone-Refractory Prostate Cancer (MDX010-21) (COMPLETED).

51. Brunet JF, Denizot F, Luciani MF, Roux-Dosseto M, Suzan M, Mattei MG, Golstein P. A new member of the immunoglobulin superfamily - CTLA-4. Nature 1987; 328: 267-70.

52. Krummel MF, Allison JP. CD28 and CTLA-4 have opposing effects on the response of T cells to stimulation. J Exp Med 1995; 182: 459-65.

53. http://www.pfizer.com/news/press-release/press-release-detail/ pfizer announces discontinuation of phase iii clinical trial for_patients_with_advanced_melanoma accessed on 10/4/2016.

54. Mechanism of Pathway: CTLA-4 Inhibition [1] https://www.azimmuno-oncology.com/ctla-4-inhibition\#Tremelimumab.

55. Poust J. Targeting metastatic melanoma. Am J Health Syst Pharm 2008; 65 (24 Suppl 9): S9-S15

56. U.S. Food and Drug Administration (September 4, 2014). "FDA approves Keytruda for advanced melanoma”. U.S. Food and Drug Administration. U.S. Food and Drug Administration. Retrieved 24 December 2015

57. Approves Anti-PD-1 Drug for Advanced Melanoma. http://www. cancernetwork.com/melanoma/fda-approves-pembrolizumab-keytruda-advanced-melanoma accessed 12/4/16.

58. US 8952136 Antibodies to human programmed death receptor PD-1. Available at: http://www.google.co.in/patents/US8952136 accessed 12/4/16. 
59. Kyi C, Postow MA. Checkpoint blocking antibodies in cancer immunotherapy. FEBS Lett. 2014; 588: 368-76.

60. Westin JR, Chu F, Zhang M, et al. Safety and activity of PD1 blockade by pidilizumab in combination with rituximab in patients with relapsed follicular lymphoma: a single group, open-label, phase 2 trial. Lancet Oncol 2014; 15: 69-77.

61. Armand P, Nagler A, Weller EA, et al. Disabling immune tolerance by programmed death-1 blockade with pidilizumab after autologous hematopoietic stem-cell transplantation for diffuse large B-cell lymphoma: results of an international phase II trial. J Clin Oncol 2013; 31: 4199-206.

62. Fairman D, Narwal R, Liang M, et al. Pharmacokinetics of MEDI4736, a fully human anti-PDL1 monoclonal antibody, in patients with advanced solid tumors. J Clin Oncol 2014; (32 suppl): abstr 2602.

63. Brahmer JR, Rizvi NA, Lutzky J, et al. Clinical activity and biomarkers of MEDI4736, an anti-PD-L1 antibody, in patients with NSCLC. J Clin Oncol 2014; (32 suppl): abstr 8021.

64. Merck-Pfizer Alliance. Merck-Pfizer Alliance Avelumab Fact Sheet (PDF). Retrieved 2 December 2015.

65. Hamid O, Robert C, Daud A, et al. Safety and tumor responses with lambrolizumab (anti-PD-1) in melanoma. N Engl J Med 2013; 369: 134-44.

66. Brahmer J, Tykodi S, Chow L, et al. Safety and activity of anti-PD-L1 antibody in patients with advanced cancer. N Engl J Med 2012; 366: 2455-65

67. Drake CG, Lipson EJ, Brahmer JR. Breathing new life into immuno therapy: review of melanoma, lung and kidney cancer. Nature Rev Clin Oncol 2014; 11: 24-37.

68. Romagné F, André P, Spee P, et al. Preclinical characterization of 1-7F9, a novel human anti-KIR receptor therapeutic antibody that augments natural killer-mediated killing of tumor cells. Blood 2009; 114: 2667-77.

69. Kirkwood JM, Lorigan P, Hersey P, et al. Phase II trial of tremelimumab $($ CP-675,206) in patients with advanced refractory or relapsed melanoma. Clin Cancer Res 2010; 16: 1042-8.

70. Hodi FS, Lee SJ, McDermott DF, et al. Multicenter, randomized phase II trial of GM-CSF (GM) plus ipilimumab (IPI) versus IPI alone in metastatic melanoma: E1608. J Clin Oncol 2013; (31 suppl): abstr CRA9007.

71. Antonia SJ, Gettinger SN, Chow LQM, et al. Nivolumab (anti-PD-1; BMS-936558, ONO-4538) and ipilimumab in first-line NSCLC: interim phase I results. J Clin Oncol 2014; (32 suppl): abstr 8023.

72. van der Most RG, Robinson BW, Lake RA. Combining immunotherapy with chemotherapy to treat cancer. Discov Med 2005; 5: 265-70.

73. Couzin-Frankel J. Breakthrough of the year 2013. Cancer immunotherapy. Science. 2013; 342: 1432-3.

\section{Address for correspondence}

Chimnoy K. Bose

NSC Bose Cancer Research Institute

8D, MSG Ln

700006 Calcutta, India

e-mail: ckbose@hotmail.com

Submitted: 29.04 .2016

Accepted: $\quad 19.12 .2016$ 